\title{
la variante souterraine, solution aux problèmes d'environnement
}

\author{
par \\ J. Brégeon \\ B. R. G. M., Orléans \\ P. Duffaut \\ B. R. G. M., Orléans
}

RESUME - Pour un grand nombre d'ouvrages, le choix d'une variante souterraine présente des avantages et des inconvénients, ceux-là l'emportent généralement sur ceux-ci en matière de protection de l'environnement. Assurément, des problèmes techniques difficiles peuvent apparâtre par exemple pour la reconnaissance géologique, et pour l'établissement du projet, qui met en jeu les mécaniques des sols, des roches et de l'eau.

Le facteur essentiel est la structure géologique : par leur disposition par rapport à la surface, et par leur structure propre, certains terrains constituent des structures préexistantes qui peuvent être très favorables pour certains usages (exemple : pièges anticlinaux pour le stockage de gaz).

C'est donc l'environnement géologique qui porte en lui-même la solution à maint problème de protection de l'environnement écologique. II y a lieu de reconnaitre les structures favorables aux usages les plus urgents, et aussi à des ouvrages plus lointains qui seraient plus contraignants ; plus généralement les Etats vont être conduits à assurer une véritable gestion de cette ressource nouvellement perçue que constitue l'espace souterrain.

\section{INTRODUCTION}

Bien des motivations ont conduit l'homme à creuser et utiliser le sous-sol, depuis la plus haute antiquité, parmi lesquelies 1'accès à l'eau souterraine, l'exploitation des ressources minérales, la conservation de denrées périssables, l'abri contre les intempéries, les bêtes sauvages ou les ennemis, sans compter des motivations plus mystérieuses d'ordre spirituel ou religieux. Dans certains cas, il n'y avait pas d'alternative : faute d'eau en surface dans les déserts, il faut aller la chercher en profondeur ; dans d'autres cas, il y avait un choix à faire entre deux variantes, soit une construction à la surface, soit un ouvrage souterrain ; quant aux exploitations minières, elles présentent aussi deux modes possibles, en découverte ou en souterrain.

La civilisation industrielle a intensifié et diversifié certaines de ces motivations ; en outre, elle en a ajouté de nouvelles en raison des besoins énergétiques, de l'extension des voies de communication et de l'accroissement considérable des surfaces bâties, tant zones urbaines que zones industrielles. La variante souterraine apparaît alors de plus en plus souvent, dans les aménagements hydroélectriques, pour le franchissement d'obstacles naturels ou artificiels, pour le stockage de produits énergétiques, mais aussi dans les innombrables réseaux et services nécessaires à la vie urbaine, enfin pour toute sorte d'activités commerciales, industrielles et militaires.

C'est aussi la civilisation industrielle qui a conduit à prendre conscience de la pollution et des nuisances et à développer le concept moderne de protection de l'environnement, en raison justement de la concentration croissante des hommes, de leurs activités, et des déchets inévitables. Si les sociétés ont cru d'abord pouvoir reléguer les activités polluantes dans des "réserves" industrielles à $1 \mathrm{a}$ façon du paysan qui relègue les porcs dans la porcherie et préserver au contraire des zones résidentielles, des zones de loisirs, voire même des zones de "canservation" de la nature, elles ont compris désormais qu'il faut appliquer partout et toujours une politique active de limitation des nuisances et des pollutions, éventuellement modulée suivant l'affectation de différentes zones.

Qubn se tienne au sens anglosaxon du terme environnement (la Terre, ses climats, ses flores et ses faunes) ou qu'on $y$ inclue au sens français tous les éléments de cadre de vie social (1'esthétique, les patrimoines historiques et culturels, les perceptions, l'hygiene et le confort, aussi bien dans le travail et le repos, dans les transports et les loisirs), il est clair que les variantes 
souterraines ne sont pas indifférentes. Dans beaucoup de cas, leurs avantages sont considérables, dans quelques uns, des inconvénients apparaissent, voire des dangers. Dans la plupart néanmoins, des problèmes techniques difficiles sont posés, qui dépendent très largement de la nature du sous-sol.

C'est donc $\ell^{\prime}$ environnement géologique qui porte en lui-même la solution à maint problème de protection de l'environnement en général. Les grandes options d'une politique d'aménagement du territoire ne doivent plus négliger les potentílités propres du soussol, éminemment variables d'un endroit à l'autre. En outre, les grandes nations seront amenées à mettre en place des systèmes de plus en plus perfectionnés de gestion de $\ell^{\prime}$ espace souterrain, dans lesquels la place de la géologie restera prépondérante.

\section{lère Partie - AVANTAGES ET INCONVENIENTS DE}

\section{LA VARIANTE SOUTERRAINE}

Il n'est guère faisable de recenser la totalité des usages possibles du sous-sol, et moins encore d'établir un tableau exhaustif des avantages et des inconvénients de la variante souterraine. Le tableau I n'a donc qu'un caractère d'exemple schématique et ne doit pas être pris comme 1'aboutissement d'une étude détaillée. Il recense trois types d'avantages et quatre types d'inconvénients :

\section{Les avantages}

Le premier avantage, c'est d'êpargner l'espace superficiel utile pour d'autres usages, urbain, industriel, agricole, etc. (il épargne aussi d'ailleurs l'espace au-dessus du sol). En effet, I'emprise des installations au sol d'une variante souterraine n'est qu'une petite fraction de l'emprise des variantes superficielles. En outre, il y a toujours de l'espace souterrain disponible et en particulier à faible distance des zones à desservir.

Le second avantage, c'est que tout ce qui est mis en souterrain est du même coup isolé, c'est-à-dire à la fois protégé contre les agressions en tout genre (ce qui ne concerne pas directement 1'environnement), mais en même temps soustrait aux regards et plus généralement soustrait à tous les sens.

La mise en souterrain des eaux usées urbaines est un exemple significatif de ce second avantage : bien avant l'ère chrétienne Rome s'est dotée d'un émissaire souterrain, Cloaca maxima. Outre le confort des sens, 1 'hygiène s'en est aussi trouvée améliorée, c'est pourquoi les systèmes d'égoûts et d'émissaires constituent aujourd'hui une des principales populations d'ouvrages souterrains dans le monde. Pour cet usage, la variante souterraine parâ̂t définitivement adoptée, favorisée il est vrai par la nécessité de recueiliir les effluents par gravité, donc au niveau le plus bas possible.

\begin{tabular}{|c|c|c|c|c|c|c|c|}
\hline \multirow{2}{*}{$\begin{array}{c}\text { Types d'utilisation } \\
\text { du sous-sol }\end{array}$} & \multicolumn{3}{|c|}{ AVANTAGES } & \multicolumn{4}{|c|}{ INCONVENIENTS } \\
\hline & $\begin{array}{l}\text { Economie } \\
\text { d'espace }\end{array}$ & Isolement & \begin{tabular}{|c|} 
Raison \\
technique
\end{tabular} & $\begin{array}{r}\text { Nuisances } \\
\text { Pollutions } \\
\end{array}$ & $\begin{array}{c}\text { Psycho et } \\
\text { Physiologiques }\end{array}$ & Juridiques & $\begin{array}{c}\text { Coût } \\
\text { Excessif }\end{array}$ \\
\hline $\begin{array}{l}\text { Extraction } \\
\text {. Eau } \\
\text {. Chaleur } \\
\text {. Pétrole } \\
\text {. Charbon } \\
\text {. Minerais } \\
\text {. Matériaux }\end{array}$ & $\begin{array}{r}x \\
x \\
x x\end{array}$ & $\begin{array}{r}\mathrm{x} \\
\mathrm{xx}\end{array}$ & $\begin{array}{r}x \\
x x \\
x x \\
x x \\
x x \\
x x\end{array}$ & $\begin{array}{r}x x \\
x \\
x\end{array}$ & $\begin{array}{r}\mathrm{xx} \\
\mathrm{x} \\
\mathrm{x}\end{array}$ & $\mathrm{x}$ & \\
\hline $\begin{array}{l}\text { Franchissement } \\
\text {. Aqueduc } \\
\text {. Chemin de fer } \\
\text {. Route }\end{array}$ & $\begin{array}{l}x \\
x \\
x\end{array}$ & & $\begin{array}{r}x \mathrm{x} \\
\mathrm{xx} \\
\mathrm{x}\end{array}$ & & & & $\mathrm{x}$ \\
\hline $\begin{array}{l}\text { Abri } \\
\text { - Denrées } \\
\text { - Denrées réfrigérées } \\
\text { - Hydrocarbures, etc. } \\
\text { - Parcsa à voitures } \\
\text { - Commerces } \\
\text { - Industries } \\
\text { - Traitement des eaux } \\
\text { - Défense }\end{array}$ & $\begin{array}{r}x \\
x \\
x x \\
x x \\
x \\
x \\
x\end{array}$ & $\begin{array}{r}x \\
x x \\
x x\end{array}$ & $\begin{array}{l}x x \\
x x\end{array}$ & $\mathrm{x}$ & $\begin{array}{r}\mathrm{xx} \\
\mathrm{x} \\
\mathrm{x}\end{array}$ & & $\mathrm{x}$ \\
\hline $\begin{array}{l}\text { Enfouissement } \\
\text { - Déchets agricoles } \\
\text { - Déchets industriels } \\
\text { - Déchets nucléaires }\end{array}$ & & $\begin{array}{l}x x \\
x x \\
x x\end{array}$ & & $\begin{array}{r}x \\
x \\
x x\end{array}$ & & $\mathrm{x}$ & \\
\hline
\end{tabular}

Tableau 1 - Principaux avantages et inconvénients de la variante souterraine

En réalitế, toutes les cases ou presque mériteraient des commentaires particuliers. Les signes $\mathrm{x}$ et $\mathrm{xx}$ indiquent des avantages ou inconvénients importants ou très importants en moyenne (sous réserve de cas particuliers). $L^{\prime}$ absence de $x$ signifie seulement moins important et non absence de lien. 
Plus généralement I'ensemble des réseaux urbains de canalisations, eau, gaz, électricité, téléphone et bien d'autres se sont enterrés peu à peu à faible profondeur. L'eau bénéficie ainsi d'une protection thermique, indispensable sous certains climats. Les autres se sont enterrés surtout pour ne pas occuper d'espace en surface ou au-dessus. C'est qu'en effet tout occupant linéaire introduit une coupure de l'espace superficiel qui peut être extrêmement gênante pour les autres usages. La suppression des coupures est un argument essentiel en faveur de l'implantation en sous-sol des canalisations, surtout les plus encombrantes, et aussi des voies ferrées. La superposition des réseaux dans 1'espace aérien, classique d'abord pour les fils et câbles, s'est étendue ensuite aux chemins de fer, puis aux autoroutes, au moins au niveau des échangeurs. Cette superposition est souvent plus facile en employant le sous-sol : les passages souterrains se multiplient au moins dans les villes, pour éviter des ouvrages bruyants au niveau des étages habités.

Un troisième avantage est d'ordre technicoéconomique, c'est d'ailleurs bien souvent celui qui a conduit d'abord aux solutions souterraines : le tunnel de circulation sous la montagne évite les dénivellations qui conduiraient au col, et les difficultés pour maintenir la voie en service l'hiver; et s'il s'agit d'un canal, il n'y a plus d'alternative, il faut passer de niveau ou abandonner ; le franchissement d'une voie maritime par dessous évite toute restriction au trafic, ainsi sous la Manche, les variantes de pont ont été écartées au profit du tunnel à cause de la vulnérabilité des piles ; la conduite forcée de l'usine hydroélectrique peut bénéficier de la résistance du terrain de même le stockage d'hydrocarbures, au-delà d'une certaine dimension, est moins cher en souterrain.

Il faut bien reconnaitre que dans la plupart des cas plusieurs avantages s'ajoutent. Si l'environnement n'a pas toujours été la première préoccupation des constructeurs d'ouvrages souterrains, il n'en est pas moins toujours bénéficiaire. De plus en plus il passe aujourd'hui au premier rang des préoccupations, ainsi dans le franchissement en souterrain d'une partie du Bois de Boulogne par le boulevard périphérique de Paris.

Quelques chiffres sommaires illustrent le gain d'espace : un volume de gaz de l'ordre du milliard de mètres cubes, tel qu'on sait le stocker dans une structure piège analogue à un gisement de pétrole, remplace plusieurs milliers de gazomètres d'autrefois (diamètre $50 \mathrm{~m}$, hauteur $40 \mathrm{~m}$ ) qui occuperaient donc ensemble une surface de plus de $10 \mathrm{~km}^{2}$. Au contraire, les installations au sol du stockage souterrain ne représentent que quelques hectares.

\section{Les inconvénients}

D'abord le danger de nuisances et pollutions, souvent cachées ou insidieuses justement parce que rien ne se voit depuis la surface. Le tableau 2 énumère divers impacts des exploitations et ouvrages dans le sous-sol, tout au moins ces impacts négatifs que sont les nuisances et les pollutions. Il comprend trois rubriques, les impacts sur la surface du sol, en position et en stabilité, sur les eaux souterraines, en niveau, débit et qualité, enfin sur le terrain lui-même, dont l'état naturel, la virginité initiale, sont irrémédiablement modifiés. La dernière rubrique, sur 1'effet de coupure, s'applique lorsqu'un usage du sous-sol empêche ou restreint notablement l'accès au sous-sol plus profond ou plus lointain.

\section{Tableau 2 - Impacts des usages du sous-sol}

I - Sur la surface du sol

affaissement vertical lent

- basculement

- affaissement brutal (mines et carrières)

. fontis

- secousses produites par injection de fluides

II - Sur l'eau souterraine

- abaissement du niveau de la nappe

- barrage sur l'écoulement de la nappe

- pollution par les effluents

- réchauffement ou congélation

- changement de pression interstitielle

III - sur le terrain lui-même

- décompression, ameublissement

- concentrations de contrainte

écoulement d'air, oxydation

- écoulement d'eau, lessivage

- occupation d'espace à 3 dimensions

- effet de coupure

Ensuite, les réactions physiologiques et psychologiques des individus appelés à travailler ou séjourner en souterrain, encore que les conditions de ce confinement soient généralement moins contraignantes que par exemple dans un sous-marin. L'expérience acquise aujourd'hui sur l'éclairage, la climatisation, la forme et la disposition des volumes est considérable, qu'il s'agisse d'installations civiles ou militaires. Il n'y a plus qu'une proportion infime de la population pour ressentir une différence gênante avec les conditions "normales".

Les difficultès juridiques ne sont pas négligeables, qui tiennent non seulement aux droits de propriété, mais aussi à l'inadaptation d'un grand nombre de réglementations. C'est seulement dans le cas des mines que les codes sont précisément adaptés au soussol. Pour les "établissements classés" comme les stockages d'hydrocarbures, la rẽglementation se développe au moins en ce qui concerne la sécurité et la pollution ; de même pour les établissements recevant du public, vis-à-vis de l'incendie et de la panique. A vrai dire, les exemples sont trop peu nombreux pour fournir une base convena- 
ble à une réglementation largement valable. Pendant longtemps encore, bien des cas resteront des cas particuliers nécessitant à la fois dérogation aux règles habituelles et imagination pour définir des règles particulières.

Enfin, le dernier domaine est celui des difficultés technico-économiques, c'est-àdire en quelque sorte le contrepoint des avantages déjà évoqués avec le même qualificatif. En effet, la variante souterraine apparaît souvent défavorisée en termes de coût et de délai d'exécution. Ce qui est le plus grave, c'est que ces deux termes sont en outre susceptibles de dépassements considérables. Certains chantiers ont duré deux fois le délai prévu, et ont coûté trois fois plus cher. Certains ouvrages n'ont pu être terminés et ont été abandonnés. Le délai de creusement des tunnels est particulièrement crucial lorsque l'accès est possible seulement par les extrémités, ainsi c'est la principale difficulté, sinon la seule, du tunnel sous la Manche, puisque ce délai se traduit par des frais financiers ajoutés au coût net. Les progrès technologiques ont considérablement amélioré l'exécution de nombreuses phases du travail. Néanmoins, la durée totale de réalisation des grands tunnels transalpins, hier ferroviaires, aujourd'hui routiers, n'a pas été réduite de manière vraiment significative.

\section{2ème Partie - PROBLEMES TECHNIQUES}

\section{Classification des problèmes}

Parmi les problèmes techniques, beaucoup ne sont pas géotechniques et ne sont donc pas abordés ci-dessous. On peut noter d'ailleurs que plusieurs se posent de la même façon pour un grand aménagement complexe, qu'il s'agisse d'un bâtiment hors sol ou enterré (exemple 1'éclairage des grands magasins aveugles, la ventilation et la protection contre l'incendie et la panique des salles de spectacles et des immeubles de grande hauteur). D'autres au contraire sont spécifiques de la variante souterraine (ventilation d'un tunnel routier, relevage des effluents, etc...).
Les problèmes géotechniques se subdivisent évidemment en fonction de nombreux facteurs, et d'abord de la nature des ouvrages à réaliser. Sans vouloir établir une classification détaillée ni même couvrir tous les cas possibles, on séparera seulement quatre classes d'ouvrages souterrains d'après leur forme générale et leur position par rapport à la surface :

- les ouvrages linéaires relativement profonds, c'est-à-dire les tunnels et les cavernes qui peuvent être considérées comme des tunnels de grande section ;

- les ouvrages superficiels, susceptibles d'être réalisés à ciel ouvert, aussi bien tranchées couvertes pour ouvrages linéaires que grandes enceintes du type de l'opération des Halles

- les ouvrages et cavités étendues à deux dimensions comme les carrières souterraines et les mines sédimentaires, avec toutefois des cas particuliers lorsque les couches ne sont pas horizontales et lorsque les cavités sont irréqulières, comme les cavités de dissolution du sel ;

- les utilisation de l'espace souterrain qui ne comportent pas d'ouvrage au sens traditionnel, sinon pour les accès, par exemple les captages d'eau souterraine, les injections de fluides, les stockages de gaz en aquifère.

Les problèmes géotechniques se subdivisent en outre suivant les spécialités à mettre en jeu. Le tableau 3 propose quatre entrées à croiser avec les classes définies ci-dessus : les reconnaissances géologiques, la mécanique des sols, la mécanique des roches, l'hydrogéologie, qui sont l'objet des chapitres ci-dessous. Un chapitre supplémentaire concerne les accès.

\section{Reconnaissances géologiques}

La première inconnue d'un ouvrage souterrain, c'est le comportement du terrain pendant les travaux.

Ce comportement proprement dit ressortit à la mécanique, mais il est largement dépendant de la nature du terrain, c'est-à-dire

\begin{tabular}{|c|c|c|c|c|}
\hline $\begin{array}{c}\text { Classes d'utilisation } \\
\text { du sous-sol }\end{array}$ & Reconnaissances & $\begin{array}{l}\text { Mécanique } \\
\text { des sols }\end{array}$ & $\begin{array}{l}\text { Mécanique } \\
\text { des roches }\end{array}$ & Hydrogéologie \\
\hline $\begin{array}{l}\text { Ia Tunnels } \\
\text { Ib Cavernes }\end{array}$ & $\begin{array}{l}\mathrm{xx} \\
\mathrm{xx}\end{array}$ & $x x$ & $\begin{array}{l}x x \\
x x\end{array}$ & $\begin{array}{l}x \\
x\end{array}$ \\
\hline II Ouvrages superficiels & $\mathrm{x}$ & $\mathrm{xx}$ & & $\mathrm{x}$ \\
\hline III Ouvrages miniers & $\mathrm{x}$ & & $\mathrm{xx}$ & $\mathrm{x}$ \\
\hline IV Pas d'ouvrages & $\mathrm{xx}$ & & & $x x$ \\
\hline
\end{tabular}

Tableau 3 - Problèmes géotechniques

(même remarques qu'au tableau 1 sur l'usage des $\mathrm{x}$ ou leur absence) 
de sa conception et de sa structure. Prévoir cette composition et cette structure, c'est l'objet des reconnaissances géologiques.

Cette prévision est relativement facile dans deux cas : celui des ouvrages miniers pourvu que $1^{\prime}$ on pense à échantillonner non seulement le minerai, mais aussi les épontes qui constitueront le mur et le toit des cavités; celui des ouvrages superficiels puisqu'il est souvent peu couteux de multiplier les tranchées et forages courts et de limiter en conséquence les distances d'interpolation.

Au contraire, cette prévision est difficile pour les tunnels profonds, et par exemple il n'est pas d'usage de faire des forages de plusieurs centaines de mètres pour étudier le tracé des tunels transalpins. C'est alors bien souvent à des hypothèses géologiques que l'on fait confiance : par exemple le granite qui apparait aux deux extrémités d'un tunnel à creuser, et en de très nombreux points sur la surface de la montagne à franchir est postulé comme un corps continu à peu près homogène (c'est en gros le cas du tunnel du Mont Blanc); ou bien la couche horizontale calcaire qui affleure sur tous les bords d'un plateau s'étend de façon continue et régulièrement horizontale sous le plateau. Ainsi, le géologue fait-il concorder avec les informations disponibles l'un des nombreux "modèles" dont il a connaissance. Du moins ne le fait-il pas au hasard, mais en se flant à des analogies de style et en étant toujours prêt à comprenare les différences.

Le rôle de la reconnaissance est toujours capital mais son utilisation comporte deux modalités : dans le cas d'un ouvrage "rigide"défini strictement à l'avance, il s'agit "seulement" de choisir le meilleur profiltype, les meilleures méthodes d'exécution, y compris les précautions éventuelles au voisinage d'une difficulté localisée, et d'estimer le coût et le délai ; fort heureusement, dans la plupart des cas, il s'agit en outre de choisir le meilleur site ou le meilleur tracé.

A cet égard, les cavernes sont favorisées parce qu'une plus grande latitude est généralement accordée au choix de leur site exact.

La reconnaissance de certaines structures est particulièrement délicate, lorsque le forage lui-même constitue un danger pour l'intégrité d'un piège (terrains salins, terrain's contenant des fluides sous pression).

\section{Mécanique des sols}

Le mot sol ne doit pas leurrer : d'une part les roches broyées le long des failles sont des sols pour le mécanicien, quelle que soit la profondeur, mais, d'autre part, les terrains meubles superficiels montrent avant remaniement des résistances plus élevées que les remblais exécutés avec le même matériau. La mécanique des matériaux de barrages en terre est souvent pessimiste, appliquée aux sols naturels en place.

Les sols sont toujours des terrains à forte porosité, donc très défavorables, même dans leur domaine élastique. C'est pourquoi les déformations autour des tunnels peuvent être considérables, et en particulier 1'affaissement de la surface du sol au-dessus du tunnel. En raison des conséquences des affaissements en milieu urbain, il s'agit d'un sujet très largement étudié, notamment dans les villes construites sur des terrains argileux. Un des exemples les plus frappants est celui des galeries de stockage de gaz proches d'Anvers à $80 \mathrm{~m}$ de profondeur.

Un important cas particulier est celui des terrains gonflants, les uns qui absorbent de l'eau parce que leur confinement naturel a été détruit, les autres grâce à des réactions chimiques avec augmentation de volume.

Le comportement parfaitement plastique est susceptible d'expliquer la stabilité de tunnels relativement profonds dans les sols, après une évolution plus ou moins importante. Les formules théoriques montrent le rôle capital du frottement pour multiplier l'efficacité du moindre soutènement. Par contre, sans soutènement, une cohésion est nécessaire, et il faut qu'elle persiste malgré les grandes déformations. En général, cette persistance n'est obtenue que grâce à un confinement.

Mais bien entendu, ce comportement favorable ne peut s'appliquer aux tunnels trop superficiels car la zone plastique rejoint la surface et le problème de sa stabilité change de nature.

\section{Mécanique des roches}

La stabilité des tunnels et cavernes en terrain rocheux est à ce point évidente qu'on n'a imaginé que récemment đe justifier le dimensionnement des éléments de soutènement provisoires ou définitifs. L'effet de voûte largement utilisé en construction au-dessus du sol, se met en oeuvre presque automatiquement, et il est favorisé dès qu'existe une composante horizontale de la contrainte initiale.

La portée d'une excavation stable en terrain rocheux est traditionnellement limitée aux environs de $25 \mathrm{~m}$; toutefois il n'y a pas d'impossibilité à envisager des portées de 50 à $60 \mathrm{~m}$, qui existent dans des grottes naturelles (maximum connu $200 \mathrm{~m}$ ) et aussi dans certaines cavités minières.

Des problèmes plus difficiles apparaissent en cas de variations de température. Ainsi 1 'échauffement jusque vers $200^{\circ} \mathrm{C}$ qui est prévu autour des dépôts de déchets nucléaires pourrait avoir des conséquences dommageables sur les roches encaissantes, fracturation supplémentaire des granites, fluage accéléré des sels gemmes par exemple. Au contraire, le refroidissement en cas de 
stockage de gaz naturels liquéfiés $\left(-161^{\circ} \mathrm{C}\right)$ doit provoquer des fissures à partir des angles de la cavité, fissures susceptibles de se propager à grande distance si le gaz liquéfié y circule sans trop s'évaporer.

\section{Hydrogéologie}

Les ouvrages souterrains qui contiennent ou véhiculent de I'eau dans un terrain non saturé sont exposés à des fuites, parfois gênantes pour d'autres usagers. Quelle que soit la situation, des effluents peuvent polluer les nappes. Mais le cas général est celui d'un ouvrage sous la nappe, qui se comporte comme un drain et récolte donc un débit, au prix d'un abaissement de la surface de la nappe. L'application de l'hydrogéologie à la prévision des débits d'exhaure transitoires et permanents est relativement classique, encore que plus difficile pour un tunnel en milieu rocheux fracturé que pour une grande foulle en alluvions.

Mais un aspect moins classique est I'effet mécanique de la pression de l'eau, surtout en l'absence de débit visible, lorsque la perméabilité du terrain est très faible, et que la pression transitoire peut se maintenir longtemps à un niveau élevé. On constate alors que la plupart des éboulements survenus au front d'avancement d'un tunnel sont plutôt des renards, et que beaucoup de gonflements de terrains argileux peuvent aussi. être attribués à cette pression.

La pression naturelle des fluides interstitiels est modifiée par de nombreuses opérations qui sont parfois susceptibles de conséquences lointaines (déjà signalées ci-dessus à titre d'impacts). Les stockages de gaz en aquifères en offrent un exemple très important, mais aussi les "manipulations" des gisements pétroliers destinés à stimuler la production : ainsi, la réinjection d'eau. Enfin, les changements de température ne sont pas à négliger : ils induisent des circulations convectives, qu'il s'agisse simplement d'extraction ou de stockage de calories, de stockages cryogéniques, ou de stockages de déchets nucléaires.

\section{Les accès}

Pour les accès aux ouvrages souterrains, la période des travaux est souvent plus contraignante que l'exploitation ultérieure. Ainsi des fenêtres ou puits intermédiaires sont ajoutés aux longues galeries hydroélectriques pour raccourcir la durée des travaux. Au contraire, pour certains tunnels routiers transalpins, des puits d'aération qui font partie du projet n'ont pas été utilisés comme accès pendant les travaux (souvent parce qu'ils prennent I'air en des sites peu accessibles).

Si l'exécution des puits ne pose pas de problème particulier (leur axe est raisonnablement incliné sur la surface du sol), celle des têtes de tunnel est d'autant plus délicate que l'angle de pénétration dans le sol est faible (tunnel horizontal dans versant à faible pente, ou bien tunnel à faible pente pénétrant un sol horizontal).

L'habitude est bien établie d'étudier avec un soin particulier les têtes de tunnels, où s'ajoutent trois difficultés : d'abord la couverture est très faible, donc aucun effet d'arc n'est mobilisable ; ensuite, le terrain superficiel est moins résistant, voire tout à fait meuble; enfin, la pente même du versant est parfois proche d'un équilibre limite que le moindre terrassement remet en cause. Heureusement, la reconnaissance est facile puisqu'elle ne porte que sur quelques mètres d'épaisseur.

\section{3ème Partie - LES STRUCTURES "CAPABLES"}

\section{Présentation}

Dans les chapitres précédents, la nature des terrains et leur structure ont été considérées d'une façon générale plutôt comme occasion de difficultés. Le géologue s'attache alors aux "défauts" du terrain, altération du granite, failles et zones broyées, en tous terrains, cavités karstiques dans le calcaire, etc.. A cette vision négative du sous-sol il y a lieu de juxtaposer une vision positive, grâce à laquelie seraient recensés au contraire les terrains favorables par leur composition et leur structure aux divers usages possibles.

Il ne s'agit d'ailleurs pas d'une démarche nouvelle puisque c'est exactement celle du mineur à la recherche du minerai ou du combustible fossile, celle aussi de l'hydrogéologue à la recherche d'eau.

\section{Les grottes}

Les grottes ont constitué de longue date des abris ou même des habitations. Pour ce premier usage, il est nécessaire que la hauteur et la largeur respectent certains gabarits. Au contraire, s'il s'agit de stocker des liquides, la section ne compte pas, mais seulement le volume et l'étanchéité. Les cavités karstiques constituent souvent une réserve d'eau potable (exemple, source du Lez près de Montpellier) et leur utilisation pour le stockage de gaz est à l'étude.

\section{Les vides artificiels}

Par rapport aux grottes, les vides abandonnés par les carrières et mines souterraines ont souvent un accès plus facile, un gabarit plus régulier et plus important, un développement plus dense qui diminue les distances d'un transport éventuel. En outre, on les trouve souvent en grand nombre au voisinage immédiat des zones urbaines.

Parmi les applications importantes, il y a lieu de citer les installations militaires de Taverny dans d'anciennes carrières de gypse, au nord-ouest de Paris, les innombrables champignonnières dans les carrières de calcaire grossier de la région parisienne, un stockage d'eau potable dans une mine dé 
fer de Lorraine et surtout le stockage de pétrole de May-sur-orne $\left(5 \mathrm{hm}^{3}\right)$.

\section{Les pièges aquifères}

La notion de piège est héritée de la géologie pétrolière. Les hydrocarbures fluides migrent dans les terrains jusqu'à ce qu'ils soient arrêtés par un piège, une structure d'où ils ne pourront plus s'échapper. Il suffit en effet d'une étanchéité à la partie supérieure. La recherche des pièges "fertiles" est l'affaire des pétroliers mais, à défaut de pétrole, un piège "stérile" représente néanmoins une importante ressource potentielle puisqu'on sait y stocker du gaz naturel. On pourrait sans doute y stocker d'autres produits, voire les y abandonner dans de bonnes conditions de sécurité.

Le recensement des pièges utilisables est donc une tâche d'inventaire d'intérêt public au même titre que par exemple l'inventaire de certaines ressources minérales.

\section{Les roches salines}

Les roches salines constituent un cas très particulier en ce qu'elles se prêtent à des cavités peu coûteuses grâce à la dissolution, qu'elles sont remarquablement étanches, et suffisamment stables.

C'est pourquoi elles ont été retenues par Gaz de France et Géostock pour le stockage de gaz naturel et de pétrole, mais aussi par l'industrie chimique pour quelques réalisations de moindre volume. La mastrise de la dissolution permet de régler la forme de la cavité.

\section{Les roches capables de grandes cavités}

Si ce titre convient déjà au sel (il existe des chambres cubiques de $60 \mathrm{~m}$ de côté dans des mines de sel de Louisiane, et des "bouteilles" obtenues par dissolution de capacité encore supérieure), il convient aussi à diverses autres roches, mais il est bien certain que des qualités particulières sont nécessaires.

En effet, ce n'est pas tellement la résistance qui compte (déjà dans la craie des cavités de grande portée sont possibles), mais c'est la continuité et l'homogénéité. En outre, il y a lieu d'adapter étroitement la forme de la cavité à la structure naturelle du terrain. Ceci est d'ailleurs un des avantages de la cavité minière sur beaucoup de cavités du génie civil : la cavité minière est obtenue par enlèvement d'une couche, le toit et le mur de la cavité sont encore des couches, c'est-à-dire des dalles.

D'une manière très générale, on peut dire qu'en terrain stratifié les cavités rectangulaires (à toit plan) sont les plus recommandées, alors qu'en terrain quasi isotrope (granite, sel gemme), les toits voutés sont préférables (à grande profondeur toute paroi plane est à éviter, comme tout angle vif).
L'adaptation à la structure peut aller plus loin que les structures visibles du terrain. En effet, suivant la valeur de la composante horizontale du tenseur contrainte, il y a lieu de modifier l'élancement de la section, en la dessinant plus élancée si la composante est négligeable, au contraire plus aplatie si la composante horizontale est plus élevée que la composante verticale.

\section{Les reliefs rocheux urbains}

Si les reliefs rocheux urbains ont été souvent exploités pour la pierre de taille (ou le gypse à Montmartre), beaucoup offrent aujourd'hui un espace souterrain doté de deux qualités rares : il est accessible de niveau tout autour ; il est naturellement drainé, ou facile à drainer par gravité. Un excellent exemple est fourni par la colline qui porte le château au centre de Salzbourg. Les pentes ont été retaillées par des exploitations de pierre de taille, puis un tunnel routier central a été creusé. Depuis peu d'années, des parcs à voitures souterrains ont été ajoutés, ainsi que des tunnels pour piétons et des galeries commerciales.

Les villes scandinaves, riches en buttes rocheuses héritées de l'érosion glaciaire (les "buttes moutonnées") y placent des stations de traitement des eaux, à l'arrivée et au départ, des abris de défense passive, dont certains utilisés comme garages en temps normal, des entrepôts variés, des salles de sport et des piscines, des arsenaux, avec des cales accessibles depuis la mer, et même une église à Helsinki.

Les entrepôts sont particulièrement précieux dans les ports des côtes rocheuses escarpées, par exemple à Bergen ou à Terre Neuve.

Il est permis d'imaginer tout ce qui pourrait être installé à l'intérieur des pains de sucre de Rio de Janeiro !

\section{Le potentiel souterrain}

En résumé, le sous-sol n'apporte pas seulement des ressources minérales (1'eau, les matériaux, les combustibles fossiles, les minerais), il apporte aussi des ressources spatiales. La connaissance et la gestion du potentiel souterrain est bien avancée pour les premières, notamment par des inventaires, des concessions, des réservations, il ne 1 'est guère pour les secondes. Et pourtant ce potentiel est aujourd'hui essentiel, non seulement pour valoriser les zones urbaines encombrées, pour préserver les zones agricoles ou de loisirs, mais aussi pour diminuer certaines dépenses énergétiques, et pour diminuer les risques inhérents à divers produits et activités industriels.

Comment évaluer ce potentiel ? Comment l'améliorer éventuellement ? (notamment dans les régions où il apparâ̂t faible en regard de la demande). L'évaluation est affaire de nombreux critères, à base de : 
- grandeurs physiques, définissant le milieu, porosité, perméabilité, surface spécifique, température, poids spécifique, chaleur massique, conductivité thermique, coefficients de dilatation, coefficients d'élasticité, résistance, etc..

- données géométriques et structurales définissant des sites, profondeur, épaisseur, directions de diaclases, de stratification, de schistosité, y compris les superpositions, les plis, les failles, etc..

- données géographiques définissant la situation par rapport aux besoins potentiels (ainsi la demande en stockages de gaz est grande au voisinage des plus grandes villes).

La figure 1 illustre schématiquement les Iimitations apportées à l'usage du sous-sol par diverses difficultés, techniques et autres, par exemple pour une tranche définie d'épaisseur du sous-sol d'une ville.

Des procédés d'exécution nouveaux feront diminuer ces limitations, mais aussi des dispositions différentes concernant les périmètres de protection, des synchronisations d'ouvrages complexes à buts multiples (par exemple l'opération des Halles).

Les mêmes raisonnements qui sont faits sur l'occupation du sol peuvent être appliqués au sous-sol. Par exemple, chaque "parcelle" à trois dimensions pourra se voir attribuer une valeur d'usage, variable suivant l'affectation. L'aménagement idéal est celui qui optimise la somme des valeurs d'usage, en tenant compte aussi bien du sol que du sous-sol.

Fig. 1 - Limitation du potentiel naturel et influence des plans de développement et d'aménagement

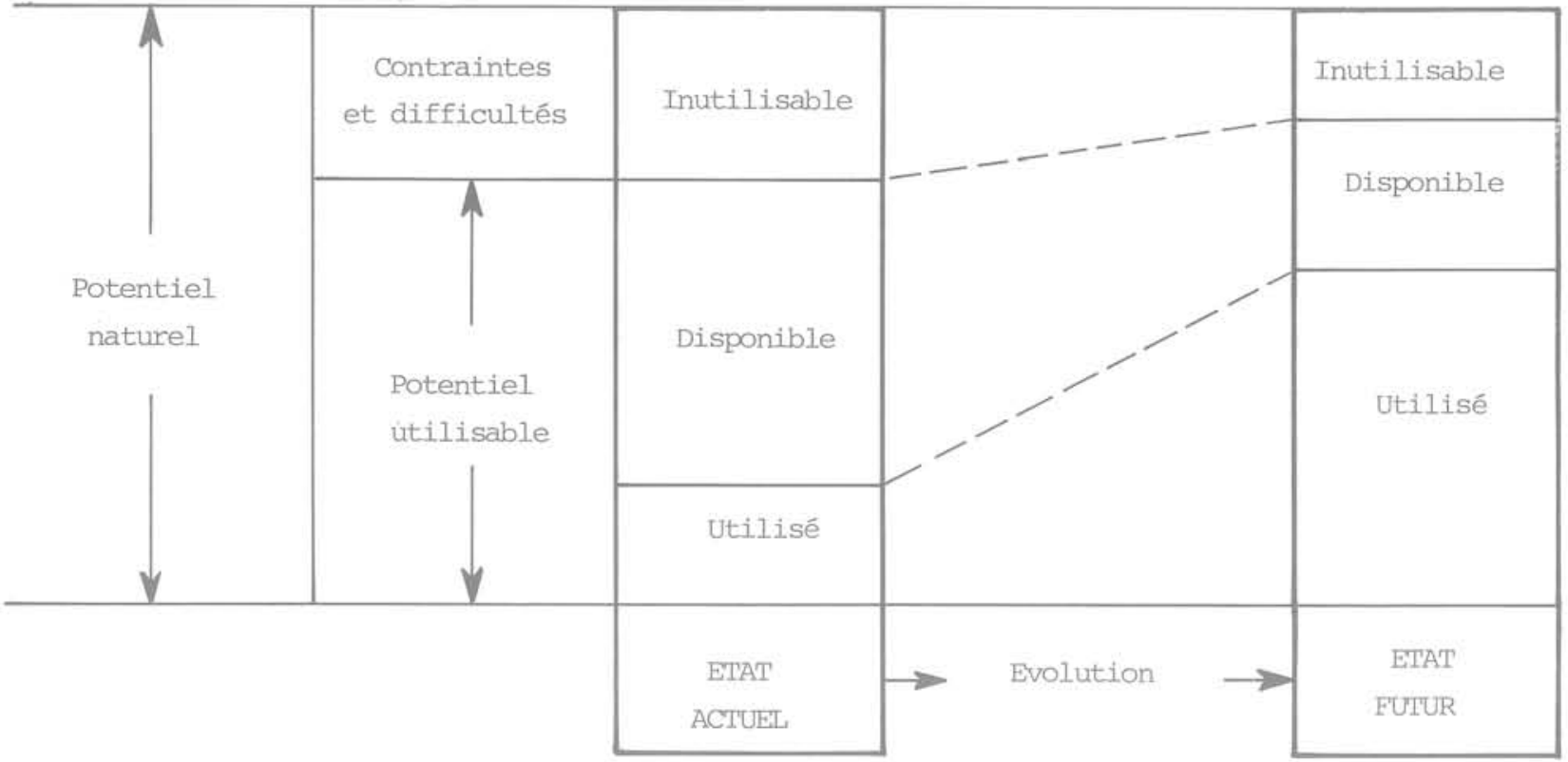

C O N C L U S I O N

Ainsi, la même société industrielle qui a, par ses excès, suscité les réactions du public puis des états en faveur d'une protection de l'environnement, apporte une réponse grâce au développement des ouvrages souterrains : lorsque le train et la locomotive sont dans le tunnel, la voie est franchissable à volonté, le bruit, les vibrations et les émissions de fumée sont supprimées. Il n'est pas étonnant que cet exemple déjà ancien soit désormais contagieux. Dans le choix entre deux variantes, le seul coût des travaux est quelquefois suffisant pour imposer le sous-sol (face notamment au coût des terrains occupés par une variante sur le sol), et lorsqu'il ne l'est pas il y a lieu d'évaluer les bénéfices sociaux qu'apporte spécifiquement la variante souterraine. Plutôt que d'optimiser le seul patrimoine d'un mâtre d'ouvrage spécialisé, il s'agit d'optimiser l'ensemble du patrimoine national.

Cet objectif ne peut être atteint que par un ensemble de démarches convergentes des différents mâtres d'ouvrages et de l'état. C'est à l'état qu'incombe la responsabilité de la gestion des ressources du sous-sol par des mesures réglementaires, des incita- 
tions, des interdictions. Pour l'assumer, il doit inventorier assez longtemps à l'avance aussi bien les besoins que les ressources ; il pourra recourir à de véritables plans d'occupation du sous-sol (POS) et définir les coefficients d'occupation (COSS), le tout à trois dimensions.

De son côté, chaque mâttre d'ouvrage se doit d'analyser de façon approfondie les avantages et les inconvénients de la variante souterraine, quitte à demander à l'état une contrepartie pour des avantages qui profiteront à d'autres (par exemple la suppression de l'effet de coupure économisera les ouvrages de franchissement d'une voie ferrée par une autoroute à construire ultérieurement).

C'est assurément le sous-sol urbain qui pose les problèmes les plus urgents, puisque les besoins y sont les plus grands, alors qu'il est déjà au moins de façon superficielle très encombré.

Mais, pour toute ville nouvelle ou grande zone industrielle à créer, et par exemple un aérodrome dont l'espace au sol et au-dessus doivent être laissés libres, il est pos- sible de confronter en temps utile les besoins, classés d'après les qualités les plus rares qu'ils attendent du milieu, et les sites classés d'après ces mêmes qualités.

Parmi les questions qui doivent être posées, figure toujours l'usage à long terme au même endroit. C'est qu'en effet il est plus difficile de modifier un ouvrage souterrain qu'un ouvrage à l'air libre. Pis encore, il est impossible de l'éliminer, alors qu'en démolissant les constructions ordinaires on rend le sol apte à de nouveaux usages, En sous-sol, rien ne peut ramener à l'état initial.

C'est donc au prix seulement de réflexions très sérieuses que l'environnement qéoloqique apportera la solution à maintproblème de protection de l'environnement écologique. Par delà la géologie traditionnelle du génie civil, c'est un nouveau chapitre de la gêologie de l'aménagement qu'un usage accru du sous-sol permettra d'écrire. 